\title{
Periodontitis and Helicobacter pylori Infection: Eradication and Periodontal Therapy Combination
}

\author{
Athanasios Tsimpiris ${ }^{1,2}$ Andreas Grigoriadis ${ }^{2,3 \odot ~ I o a n n i s ~ T s o l i a n o s ~}{ }^{4}$ \\ Ioannis Moschos ${ }^{5}$ Dimitrios G. Goulis ${ }^{6}$ Georgios Kouklakis ${ }^{7}$
}

${ }^{1}$ Department of Medicine, Democritus University of Thrace, Alexandroupolis, Greece

${ }^{2}$ Dental Sector, 424 General Military Training Hospital, Thessaloniki, Greece

${ }^{3}$ Department of Preventive Dentistry, Periodontology and Implant Biology, Dental School, Faculty of Health Sciences, Aristotle

University of Thessaloniki, Thessaloniki, Greece

${ }^{4}$ Dental School, Faculty of Health Sciences, Aristotle University of Thessaloniki, Thessaloniki, Greece

${ }^{5}$ Department of Nursing, International Hellenic University, Thessaloniki, Greece

6 Unit of Reproductive Endocrinology, First Department of Obstetrics and Gynecology, Medical School, Aristotle University of Thessaloniki, Thessaloniki, Greece

${ }^{7} A^{\prime}$ Department of Pathology, Department of Medicine, Democritus University of Thrace, Alexandroupolis, Greece

\author{
Address for correspondence Athanasios Tsimpiris, DDS, MSc, PhD, \\ Dental Sector, 424 General Military Training Hospital. 1-3 Grigoriou \\ Lampraki Street, 54636 Thessaloniki, Greece \\ (e-mail: atsimpir@gmail.com).
}

Eur J Dent 2022;16:145-152.

\begin{abstract}
Keywords

- Helicobacter pylori

- periodontitis

- saliva

- oral cavity

- eradication

- periodontal therapy

Objectives This study was aimed to assess (1) the prevalence of salivary and gastric Helicobacter pylori (HP) infection in patients with and without periodontitis, (2) the prevalence of HP infection in patients with periodontitis according to its clinical classification, (3) the effect of periodontitis treatment in patients with or without gastric HP infection, and (4) if gastric HP eradication in combination with periodontitis treatment influences periodontitis clinical outcome.

Materials and Methods Thirty-three adults with periodontitis treated by quadrant scaling and root planning (QSRP). The simplified plaque index (PI), bleeding on probing index (BOP), probing pocket depth (PPD), and clinical attachment level (CAL) were assessed pretreatment and 3 months of posttreatment. The patients were tested for oral and gastric HP. Gastric HP (+) patients received eradication therapy. A control group of 32 periodontically healthy volunteers was tested for oral and gastric HP. Saliva samples were evaluated by real-time polymerase chain reaction (rtPCR); gastric HP was detected by urea breath test (UBT).

Statistical Analysis Normality of variables assessed by the Kolmogorov-Smirnov test, while the differences of pre- and post-treatment were analyzed by paired samples $t$-test. Differences between subgroups were compared by a Student's $t$-test or a Mann-Whitney $U$-test. Comparisons of nominal variables were made by Pearson's Chi-square test.

Results No saliva samples were positive for HP. Gastric HP was detected in six patients with periodontitis and seven controls $(p>0.05)$. HP infection affected patients with higher disease stages and grades. All HP (+) patients underwent successful eradication treatment. All clinical periodontitis indices improved following QSRP. HP (+) patients who received QSRP as adjunctive to eradication treatment showed improvement in

published online

October 1, 2021

DoI https://doi.org/ $10.1055 / \mathrm{s}-0041-1731928$ ISSN $1305-7456$

(c) 2021. The Author(s).

This is an open access article published by Thieme under the terms of the Creative Commons Attribution License, permitting unrestricted use, distribution, and reproduction so long as the original work is properly cited. (https:// creativecommons. org/licenses/by/4.0/).

Thieme Medical and Scientific Publishers Pvt. Ltd. A-12, 2nd Floor, Sector 2, Noida-201301 UP, India
\end{abstract}


BOP $(p<0.001)$, PI $(p<0.013)$, and CAL $(p<0.004)$ compared with HP $(-)$ patients who received QSRP alone.

Conclusion Periodontitis was not associated with gastric HP infection. Saliva was not a gastric HP supply reservoir. Gastric HP infection was associated with advanced stages and grades of periodontitis. Although all periodontal clinical markers improved after QSRP, BOP, PI, and CAL, they were further improved when combined with eradication treatment. Periodontal evaluation and treatment combined with HP eradication are recommended in patients with HP gastric infection.

\section{Introduction}

Advanced periodontitis is the sixth commonest disease worldwide, with a prevalence of 10.8 to $11.2 \%{ }^{1,2}$ It is a chronic inflammatory disease of the periodontal tissues due to anaerobic gram-negative bacteria ${ }^{3}$ that is characterized by progressive alveolar bone destruction, resulting in the formation of a periodontal pocket and gum retraction. ${ }^{4}$ Periodontal disease can be considered to disrupt homeostatic mechanisms that balance the oral biofilm microflora and the host defense mechanisms. ${ }^{5}$ Periodontitis has been associated with cardiovascular disease, certain types of cancer, type-2 diabetes, and pregnancy complications. ${ }^{6}$

Helicobacter pylori (HP) is a microaerophilic gram-negative spiral-helical bacterium adapted to survive in the gastric acidic environment, neutralizing it by secreting urease. ${ }^{7}$ A higher prevalence of gastric HP infection exists in developing countries (50.8\%) compared with the developed ones (34.7\%). ${ }^{8}$ Gastric mucosal infection by HP has been associated with gastritis, gastric and duodenal ulcers, and gastric cancer. ${ }^{9}$ In addition, HP infection has been associated with several nonpeptic diseases, such as coronary heart disease and cardiovascular disease, diabetes mellitus, and anemia. ${ }^{10}$ Also, HP infection has been associated with oral cavity diseases, such as recurrent aphthous stomatitis, ${ }^{11}$ halitosis, burning mouth syndrome, lingual hyperplasia, ${ }^{12}$ and oral lichen planus. ${ }^{13}$

Treatment of HP infection is by combinations of antibiotics and proton pump inhibitors (PPI), taken simultaneously or sequentially for 7 to 14 days; however, in clinical practice, no treatment guarantees HP eradication. ${ }^{14}$ The high rates of HP infection recurrence led researchers to investigate whether the oral cavity is an extra-gastric HP reservoir, as systemic antimicrobials against HP fail to disrupt the germ-protective oral biofilm. ${ }^{15}$ Studies demonstrated the permissive role of chronic periodontal disease in the colonization of the oral cavity and gastric infection by HP. ${ }^{16}$

The aims of the present study were (1) to assess the prevalence of HP infection in patients with and without periodontitis; (2) to assess the prevalence of HP infection in patients with periodontitis according to the clinical classification of the latter; (3) to evaluate the effect of periodontitis treatment in patients with or without gastric HP infection; and (4) to evaluate if gastric HP eradication treatment in patients with HP infection treatment in combination with periodontitis treatment influences periodontitis clinical outcome.

\section{Materials and Methods}

\section{Patients}

Thirty-three patients attended the hospital's dental sector from April 2019 to October 2020 and were diagnosed with periodontitis stage $\geq 2 .{ }^{17}$ Thirty-two periodontically healthy volunteers, matched for sex and age to the patients, served as a control group. Exclusion criteria were age $<18$ years, previous HP eradication attempt, use of antibiotics, bismuth compounds, PPIs, $\mathrm{H}_{2}$ blockers, or antacids within the last 2 months, pregnancy, diabetes, immune diseases, immunosuppression for any cause, chronic use of nonsteroidal anti-inflammatory drugs (NSAIDs), history of gastric surgery, malignancies, periodontal treatment in the last 6 months, and $<18$ natural teeth.

\section{Protocol}

The periodontal examination was performed at six sites of each tooth (mesiobuccal, midbuccal, distobuccal, mesiolingual, midlingual, and distolingual) and included for each site as follows: probing pocket depth (PPD; distance from the gingival margin to the bottom of the gingival sulcus, in $\mathrm{mm}$ ), evaluation of clinical attachment level (CAL; distance from the cementoenamel junction to the bottom of the gingival sulcus, in $\mathrm{mm}$ ), simplified plaque index (PI; presence or absence of supragingival plaque using basic fuchsin as a disclosing agent), and presence or absence of bleeding on probing (BOP) 30 seconds after PPD. An orthopantomography was performed in all patients for radiographic evaluation of the jawbone. Patients with PPD $\geq 4 \mathrm{~mm}$ and/or CAL $\geq 4 \mathrm{~mm}$ $>30 \%$ of the measurement surfaces and BOP $>10 \%$ were classified into the test group ( $n=33$ ), while those with BOP $<10 \%$ and PPD $\leq 3 \mathrm{~mm}$ were classified into the control group ( $n=$ 32). All measurements were made using a manual periodontal probe (University of North Carolina - 15, Hu-Friedy) by the same experienced periodontist (A.G.). The intraexaminer error was high (kappa $>0.80$ ), and the measurements had $>90 \%$ agreement for $\pm 1 \mathrm{~mm}$ and exact agreement in $>75 \%$ of the PPD repeated measurements. ${ }^{18}$ The presence of HP in saliva and stomach in both groups was detected by real-time polymerase chain reaction (rtPCR) and urea breath test (UBT) method, respectively. 
During a second visit, a saliva sample was collected, and the detection of gastric HP was performed by UBT. All patients arrived in the morning without eating for $>6$ hours and without brushing their teeth or rinsing their mouths. The saliva was collected by a sterile swab with a synthetic fiber tip to wipe the inside of the cheeks and placed in a sterile 2-ml Eppendorf with $500 \mu \mathrm{L} \mathrm{NaCl} 0.9 \%$; it was stored at 2 to $8^{\circ} \mathrm{C}$ for 18 to 24 hours or at $-20^{\circ} \mathrm{C}$ for up to 3 months, depending on the test time.

\section{Real-Time Polymerase Chain Reaction}

The VIASURE HP rtPCR Detection Kit (CERTEST BIOTEC, Spain, CE-IVD) was used for the molecular detection of HP which was evaluated against strain J99, targeting in virulence gen ureB that encodes one of bacterial urease's structural subunit. DNA extraction was performed by the PREP-NADNA/RNA Extraction Kit, DNA Technology, CE-IVD. The VIASURE kit is based on the five-exonuclease activity of DNA polymerase. The fluorescence was measured by Stratagene mx3005p (Agilent, United States).

\section{Urea Breath Test}

The Helicobacter test INFAI $75 \mathrm{mg}{ }^{13} \mathrm{C}$ was used. Two breath samples were collected by blowing through a straw into two glass tubes with a stopper. Subsequently, each patient received $200 \mathrm{~mL}$ of natural orange juice to delay gastric emptying and ingested a drink containing $75 \mathrm{mg}{ }^{13} \mathrm{C}$-marked urea $(30 \mathrm{~mL})$. After 30 minutes, the blowing exercise was repeated to collect postdose samples. The presence of gastric HP leads to hydrolysis of urea by the enzyme urease and release of marked ${ }^{13} \mathrm{CO}_{2}$. The four breath samples were sent to a central laboratory for ${ }^{13} \mathrm{C} /{ }^{12} \mathrm{C}$ ratio analyses on exhaled $\mathrm{CO}_{2}$ by mass spectrometry. HP infection was detected if the difference in ${ }^{13} \mathrm{C} /{ }^{12} \mathrm{C}$ ratio between the pre- and post- ${ }^{13} \mathrm{C}$ samples were $>4.0 \%$. UBT presents high sensitivity (>95\%) and specificity $(>93 \%){ }^{19}$

\section{Treatment}

All patients in the test group were treated with quadrant scaling and root planning (QSRP) under local anesthesia (4\% articaine hydrochloride with epinephrine $1: 100,000$ ) in four appointments, with an interval of 1 week between them and reexamined after 3 months. The instruments used were periodontal curettes (Gracey Access curettes, Kohler, Austria) and ultrasonic scalers (Piezon 250, EMS, Switzerland). In gastric HP $(+)$ patients, sequential therapy was administered for 10 days (dual therapy including a PPI plus amoxicillin 1-g twice daily both, for the first 5 days followed by a triple therapy including a PPI, clarithromycin of $500 \mathrm{mg}$ and metronidazole of 500-mg twice daily all, for the remaining 5 days) immediately after the end of QSRP. Three months after QSRP, measurements of clinical periodontal markers were performed by the same periodontist who performed the initial examination, not knowing which patients received antibiotic treatment and by the same manual periodontal probe. The efficacy of gastric HP eradication in HP $(+)$ patients from the experimental group was also tested by UBT, 3 months after QSRP. The study protocol and timeline diagram it is shown in - Fig. 1.

\section{Preliminary Power Analysis}

As a preliminary power test, assuming a mean PPD difference of $2 \mathrm{~mm}$ with a pooled standard deviation of $1 \mathrm{~mm}$, we would require six patients in our gastric $\mathrm{HP}(+)$ sample to achieve power exceeding the 0.8 rule-of-thumb $(0.876) .{ }^{20}$

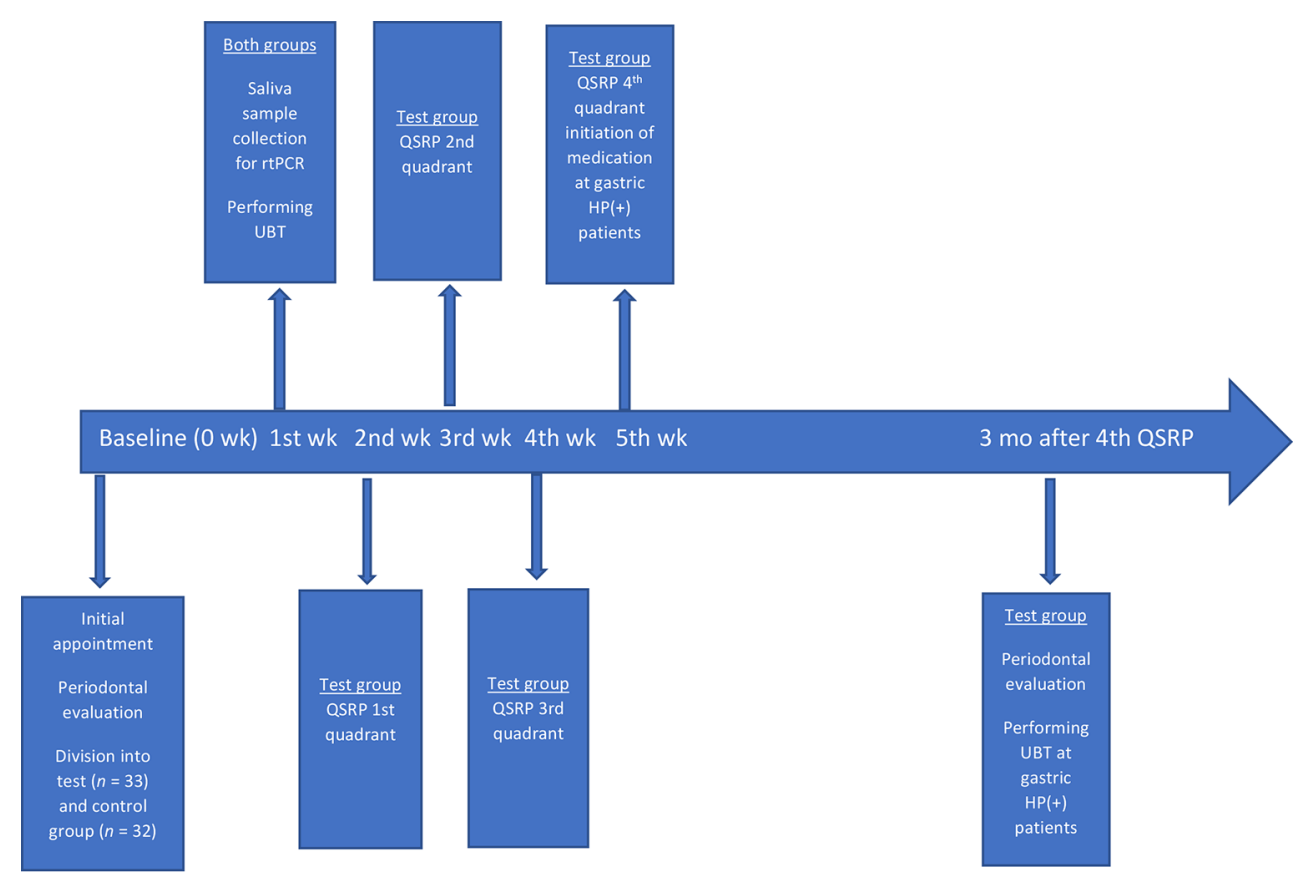

Fig. 1 Timeline and study protocol diagram. HP, Helicobacter pylori; QSRP, quadrant scaling and root planning; rtPCR, real-time polymerase chain reaction; UBT, urea breath test. 


\section{Statistical Methods}

The normality of variables (BOP, PI, PPD, and CAL) was assessed by the Kolmogorov-Smirnov test statistic. The statistical comparisons were performed within the test group by dividing it into two subgroups, those with and without HP, since the control group did not receive any treatment. The differences in study variables, pre- and posttreatment were analyzed by paired samples $t$-test; the differences between HP (+) and HP (-) subgroups were compared by a Student's $t$-test or a Mann-Whitney $U$-test, depending on whether they were distributed normally or not. Comparisons of nominal variables and percentages were made by Pearson's Chi-square test. A $p$-value of $<0.05$ was considered statistically significant. The analysis was performed using the IBM SPSS, version 21.0 for Windows.

\section{Results}

\section{Patient Matching}

No differences were observed between the patient and control groups regarding sex (14/33 and 14/32 males, respectively, Chi-square, $p=0.914)$ or age (55.5 \pm 12.4 and $55.5 \pm$ 13.4 years, respectively, $t$-test [62.22], $p=0.944$ ).

\section{Prevalence of Helicobacter pylori Infection in Patients with and without Periodontitis}

HP was not detected in the saliva by PCR in any patient or control group, but it was detected in the stomach of seven controls and six patients (Chi-square: $\chi^{2}(1)=0.138, p=0.71$ ).

\section{Prevalence of Helicobacter pylori Infection according to Periodontitis Classification \\ No differences in periodontitis stages or grades were observed posttreatment. $\mathrm{HP}(+)$ patients presented with higher disease severity for staging (Chi-square $=11.873, p=0.003$ ) and grad- ing (Chi-square $=7.792, p=0.02 ;$ - Table 1 ).}

\section{Periodontitis Treatment in Patients with or without Gastric Helicobacter pylori Infection}

All patients with HP underwent successful eradication treatment. Measurements of pre- and posttreatment BOP, PI, PPD, and CAL in patients with and without gastric HP infection are presented in - Table $\mathbf{2}$. All patients improved with treatment, with a comparative benefit for patients who had HP (+). Benefits in CAL for both groups were the smallest, with very little benefit in HP (+) patients and no benefit for HP (-) patients. The PPD was decreased by approximately $1.3 \mathrm{~mm}$ in $\mathrm{HP}(+)$ patients and by approximately $0.9 \mathrm{~mm}$ in $\mathrm{HP}(-)$ patients.

\section{Combination of Eradication Treatment and Quadrant Scaling and Root Planning}

- Table 3 presents the comparisons of differences in preand posttreatment POB, PI, PPD, and CAL in patients with and without gastric HP infection. Periodontal debridement provided additional benefit for patients with HP who undergo treatment for the infection compared with HP (-) patients.

\section{Discussion}

The role of the oral cavity, especially when periodontitis is present, in the transmission and recurrence of HP infection is a subject of discussion and considerable divergence. In the present study, HP was not detected in the saliva of patients with periodontitis or healthy patients, regardless of gastric HP infection. The result is consistent with previous studies, in which HP was not detected in the saliva of 49 patients with dyspepsia ${ }^{21}$; the subgingival plaque of 115 patients $^{22}$; the supra- or subgingival plaque of 62 patients with gingivitis, mild and moderate periodontitis ${ }^{23}$; or the saliva, tongue, and dental plaque of 43 patients with gastric disorders. ${ }^{24}$ A large study did not detect HP in 1,000 periodontal pockets of 336 adult patients with periodontitis, ${ }^{25}$ while a $3.4 \%$ prevalence of HP positivity was detected in the saliva of 58 clinically healthy volunteers. ${ }^{26} \mathrm{~A}$ recent study did not find an association between periodontitis and the presence of HP in dental plaque of 50 patients with periodontitis and 50 controls. ${ }^{27}$ On the other hand, high prevalence of HP in saliva (55\%) and dental plaque (97\%) and lower ones in the stomach (26.2\%) led to the opinion that HP belongs to the normal oral flora, ${ }^{28}$ or the oral cavity serves as a reservoir for gastric HP infection. ${ }^{29}$ Other authors argue that HP exists in the oral cavity only as a transient organism, as other competing species colonize and predominate. ${ }^{30}$ The transient presence of HP in the oral cavity has been attributed to the contamination of the latter by gastric fluid due to reflux. ${ }^{23,29}$ The

Table 1 Patients staging and grading pretreatment

\begin{tabular}{|l|l|l|l|l|}
\hline \multicolumn{2}{|l|}{ Periodontitis classification } & \multicolumn{2}{l|}{ Helicobacter pylori in stomach } & \multirow{2}{*}{ Total } \\
\cline { 2 - 5 } \multicolumn{2}{|l|}{} & Yes & No & 6 \\
\hline Pretreatment stage & 2 & 0 & 6 & 21 \\
\cline { 2 - 5 } & 3 & 2 & 19 & 6 \\
\cline { 2 - 5 } & 4 & 4 & 2 & 2 \\
\hline Pretreatment grade & 1 & 0 & 2 & 15 \\
\cline { 2 - 5 } & 2 & 0 & 15 & 16 \\
\cline { 2 - 5 } & 3 & 6 & 10 & \\
\hline
\end{tabular}


Periodontitis and Helicobacter pylori Infection Tsimpiris et al. 149

Table 2 Measurements of pre- and post-treatment BOP, PI, PPD, and CAL in patients with and without gastric Helicobacter pylori infection

\begin{tabular}{|c|c|c|c|c|c|c|c|c|}
\hline $\begin{array}{l}\text { Gastric } \\
\text { H. Pylori } \\
\text { detection }\end{array}$ & $\begin{array}{l}\text { Periodontitis clinical } \\
\text { markers }\end{array}$ & Mean & SD & $\begin{array}{l}\text { Mean } \\
\text { difference }\end{array}$ & SD & $\begin{array}{l}t \text {-test (paired } \\
\text { samples) }\end{array}$ & $p$-Value & Effect size (d) \\
\hline \multirow[t]{8}{*}{ Yes $(n=6)$} & BOP pretreatment (p/tp) & 0.814 & 0.224 & \multirow[t]{2}{*}{0.674} & \multirow[t]{2}{*}{0.203} & \multirow[t]{2}{*}{$8.112, d f=5$} & \multirow[t]{2}{*}{$<0.001$} & \multirow[t]{2}{*}{3.311} \\
\hline & BOP posttreatment ( $\mathrm{p} / \mathrm{tp})$ & 0.139 & 0.051 & & & & & \\
\hline & PI pretreatment (p/tp) & 0.720 & 0.289 & \multirow[t]{2}{*}{0.596} & \multirow[t]{2}{*}{0.308} & \multirow[t]{2}{*}{$4.742, \mathrm{df}=5$} & \multirow[t]{2}{*}{0.005} & \multirow[t]{2}{*}{1.935} \\
\hline & PI posttreatment (p/tp) & 0.124 & 0.043 & & & & & \\
\hline & PPD pretreatment (mm) & 3.843 & 1.721 & \multirow[t]{2}{*}{1.278} & \multirow[t]{2}{*}{0.633} & \multirow[t]{2}{*}{$4.938, d f=5$} & \multirow[t]{2}{*}{0.004} & \multirow[t]{2}{*}{2.018} \\
\hline & PPD posttreatment (mm) & 2.565 & 1.109 & & & & & \\
\hline & CAL pretreatment (mm) & 4.403 & 1.901 & \multirow[t]{2}{*}{0.183} & \multirow[t]{2}{*}{0.257} & \multirow[t]{2}{*}{$1.748, \mathrm{df}=5$} & \multirow[t]{2}{*}{0.141} & \multirow[t]{2}{*}{0.712} \\
\hline & CAL posttreatment $(\mathrm{mm})$ & 4.220 & 1.850 & & & & & \\
\hline \multirow[t]{8}{*}{ No $(n=27)$} & BOP pretreatment (p/tp) & 0.502 & 0.281 & \multirow[t]{2}{*}{0.303} & \multirow[t]{2}{*}{0.191} & \multirow[t]{2}{*}{$8.232 \mathrm{df}=26$} & \multirow[t]{2}{*}{$<0.001$} & \multirow[t]{2}{*}{1.584} \\
\hline & BOP posttreatment ( $\mathrm{p} / \mathrm{tp})$ & 0.199 & 0.119 & & & & & \\
\hline & PI pretreatment (p/tp) & 0.388 & 0.266 & \multirow[t]{2}{*}{0.223} & \multirow[t]{2}{*}{0.195} & \multirow[t]{2}{*}{$5.945, d f=26$} & \multirow[t]{2}{*}{$<0.001$} & \multirow[t]{2}{*}{1.144} \\
\hline & PI posttreatment (p/tp) & 0.164 & 0.119 & & & & & \\
\hline & PPD pretreatment (mm) & 3.608 & 0.837 & \multirow[t]{2}{*}{0.905} & \multirow[t]{2}{*}{0.365} & \multirow[t]{2}{*}{$12.889, \mathrm{df}=26$} & \multirow[t]{2}{*}{$<0.001$} & 2.479 \\
\hline & PPD posttreatment (mm) & 2.702 & 0.594 & & & & & \\
\hline & CAL pretreatment (mm) & 4.217 & 1.271 & -0.003 & 0.027 & $-0.723, \mathrm{df}=26$ & 0.476 & 0.111 \\
\hline & CAL posttreatment $(\mathrm{mm})$ & 4.221 & 1.258 & & & & & \\
\hline
\end{tabular}

Abbreviations: BOP, bleeding on probing; CAL, clinical attachment level; df, degrees of freedom; p/tp, points/total points; PI, plaque index; PPD, probing pocket depth; SD, standard deviation.

Table 3 Comparisons of differences in pre- and posttreatment POB, PI, PPD, and CAL in patients with and without gastric Helicobacter pylori infection

\begin{tabular}{|c|c|c|c|c|c|c|c|c|}
\hline $\begin{array}{l}\text { Periodontitis } \\
\text { clinical } \\
\text { markers }\end{array}$ & $\begin{array}{l}\text { H. pylori in } \\
\text { stomach }\end{array}$ & $n$ & Mean & SD & $\begin{array}{l}t \text {-test }(t) / \\
\text { Mann-Whitney } \\
(z)\end{array}$ & $p$-Value & Improvement (\%) & Effect size (d) \\
\hline \multirow{2}{*}{$\begin{array}{l}\text { Difference in } \\
\text { BOP (p/tp) }\end{array}$} & Yes & 6 & 0.674 & 0.203 & \multirow[t]{2}{*}{$t=4.251$} & \multirow[t]{2}{*}{$<0.001$} & 82.18 & \multirow[t]{2}{*}{1.919} \\
\hline & No & 27 & 0.303 & 0.191 & & & 58.04 & \\
\hline \multirow{2}{*}{$\begin{array}{l}\text { Difference in } \mathrm{Pl} \\
(\mathrm{p} / \mathrm{tp})\end{array}$} & Yes & 6 & 0.596 & 0.308 & \multirow[t]{2}{*}{$z=2.474$} & \multirow[t]{2}{*}{0.013} & 76.72 & \multirow[t]{2}{*}{1.714} \\
\hline & No & 27 & 0.223 & 0.195 & & & 52.69 & \\
\hline \multirow{2}{*}{$\begin{array}{l}\text { Difference in } \\
\text { PPD (mm) }\end{array}$} & Yes & 6 & 1.278 & 0.633 & \multirow[t]{2}{*}{$t=1.965$} & \multirow[t]{2}{*}{0.060} & 33.13 & \multirow[t]{2}{*}{0.887} \\
\hline & No & 27 & 0.905 & 0.365 & & & 24.43 & \\
\hline \multirow{2}{*}{$\begin{array}{l}\text { Difference in } \\
\text { CAL (mm) }\end{array}$} & Yes & 6 & 0.183 & 0.257 & \multirow[t]{2}{*}{$z=2.918$} & \multirow[t]{2}{*}{0.004} & 6.60 & \multirow[t]{2}{*}{0.805} \\
\hline & No & 27 & 0.003 & 0.027 & & & 0.22 & \\
\hline
\end{tabular}

Abbreviations: BOP, bleeding on probing; CAL, clinical attachment level; p/tp, points/total points; PI, plaque index; PPD, probing pocket depth; SD, standard deviation.

transient presence of oral HP is supported by high immunoglobulin (Ig)-A concentrations detected in the saliva of 100 adult women with and without periodontitis, regardless of HP detection in the mouth. ${ }^{31}$ Also, in the present study, there was no direct correlation between gastric HP infection and periodontal condition regardless of the presence of HP in the oral cavity. There is disagreement in the literature on this issue, as some researchers agree with this result ${ }^{32}$ and some do not..$^{33}$ The comparison of all results of the studies named above presents difficulties, as they have applied different methodological procedures (primers, sampling methods, and protocols) ${ }^{34}$ on different populations. ${ }^{15}$ The possibility of different HP genotypes in the saliva, stomach, and stools of the same person adds to the diagnostic difficulty, ${ }^{35}$ as PCR cross-sensitivity with other Helicobacter strains ${ }^{34}$ and false-positive results from dead bacteria. ${ }^{36}$

The prevalence of HP in the saliva is lower than those of dental plaque, regardless of the measurement method,,$^{15}$ as factors, such as HP adhesion to biofilm, saliva flow, ${ }^{37}$ and its antimicrobial content, ${ }^{24}$ may reduce the detectable microbial load. Salivary gland secretions, viruses, fungi, and epithelial cells, ${ }^{36}$ the changing $\mathrm{pH}^{38}$ and the time of sampling, due to the changing salivary flow during the day, may cause detection problems. ${ }^{36}$

In the present study, there was $100 \%$ efficacy of gastric HP eradication, in accordance with the literature. In a 
meta-analysis of seven randomized controlled trials (RCTs), including 691 participants, periodontal treatment combined with eradication treatment increased the rate of gastric HP eradication compared with eradication therapy alone. ${ }^{39}$ In another meta-analysis, eradication treatment is more effective in the recurrence of gastric HP, combined with periodontal debridement than alone. ${ }^{40}$ Gastric HP eradication occurred at a lower rate compared with the present study (87.4 vs. $100 \%$ ), 3 months after triple treatment without periodontal therapy, in patients who were $\mathrm{HP}(+)$ in the stomach and $\mathrm{HP}(-)$ in the oral cavity. ${ }^{41}$

Although the present study did not find an association between gastric HP infection and periodontitis, it showed an association between gastric HP infection and the severity of periodontal disease, regardless of HP detection in saliva. HP was not detected in the stomach of patients with stage- 2 periodontitis, while the prevalence was 9.5 and $66.6 \%$ for stages 3 and 4, respectively. Scarce evidence is available on this association, as most research focuses on the association between periodontitis independently of its severity and gastric HP infection. In a recent study, 39.4\% of patients with mild periodontitis were diagnosed with HP infection, while this prevalence rose to 70 and $85.7 \%$ in patients with moderate and advanced periodontitis, respectively ${ }^{42}$ Another study ${ }^{43}$ found a positive association between gastric HP infection and the number of lost teeth as a proxy of advanced periodontal disease. The detection of HP only in moderate-to-severe periodontal pockets ${ }^{16}$ supported this association. Adachi et al measured lactate dehydrogenase (LDH) and hemoglobin $(\mathrm{Hb})$ concentrations in saliva to diagnose the degree of periodontal tissue damage and the degree of bleeding gums, respectively, in 686 patients, and showed that gastric HP infection is a potential risk factor for the onset and deterioration of periodontal disease. ${ }^{44}$

The association between gastric HP infection and advanced grade of periodontal disease is another finding of the present study, as all HP (+) patients grade to the rapid rate of periodontitis progression (grade $\mathrm{C}$ ). As the grades "reflect biologic features of the disease including evidence of, or risk for, rapid progression, anticipated treatment response, and effects on systemic health,"17 this finding could partly explain the improvement and the high-to-moderate effect sizes of HP (+) patients who received eradication in addition to periodontal treatment. As the present study did not attempt to detect HP in other parts of the oral cavity, where the microorganism could be present, antibiotic treatment may have helped eliminate the hidden HP that could potentially cause rapid progression and/or poor response of periodontitis to previous treatments. Thus, while all patients benefited from QSRP, those receiving adjuvant HP eradication treatment showed an improvement in most periodontal clinical markers (BOP, PI, and CAL) compared with those HP (-) patients who did not receive eradication treatment. Perhaps, the relatively small sample size did not allow PPD to achieve statistical significance. However, according to much of the literature, greater clinical improvement is reported for patients with periodontitis, receiving both periodontal treatment and systemic antibiotics compared with those who receive periodontal treatment alone, regardless of the presence of HP. ${ }^{45}$ Thus, the lack of a group of HP (+) patients with periodontal disease who received only periodontal treatment without antibiotic therapy constitutes a limitation of this study, given the complexity of the pathogenic mechanisms involved in periodontal disease, it cannot be claimed that the further improvement of the periodontal status is a direct result of the medication.

As HP was not detected at root canals of teeth with asymptomatic apical periodontitis and aspirates from acute apical abscesses, it seems that the necrotic root canal does not serve as an HP reservoir. ${ }^{46}$ On the contrary, it seems that HP infection may be indirectly related to periodontitis, even its advanced forms, through periodontal disease bacteria only, such as Campylobacter rectus and Tannerella forsythia, ${ }^{30,47,48}$ which can compete and bind HP strains. This binding, which might be the reason for the temporality of HP in the mouth, may lead to a cross-antigenicity of HP and periopathogens through heat-shock proteins, resulting in increased inflammatory immune response in the stomach and the mouth. ${ }^{30,47}$

Associating gastric HP infection with the stages and degrees of periodontitis, rather than periodontitis as a dichotomous variable, is an advantage of the present study. However, the small sample size and the study type (single center) constitute disadvantages. Further research is needed on larger populations of patients and controls to draw safe conclusions.

\section{Conclusion}

In conclusion, the present study indicates that periodontitis is not directly related to gastric HP infection, neither is saliva a supply reservoir of gastric HP. However, there is an association of HP gastric infection with advanced stages and grades of periodontitis when these two conditions coexist. Although the periodontal clinical markers improved after QSRP in all patients, regardless of HP gastric infection, BOP, PI, and CAL improved more in patients who underwent HP eradication compared with those who underwent QSRP only; thus, periodontal evaluation and treatment combined with HP eradication in gastric $\mathrm{HP}(+)$ patients are recommended.

\section{Ethical Approval}

The study protocol was approved by the Ethics Committee of Democritus University of Thrace and by the Scientific Research Ethical Committee of 424 Military Hospital (Thessaloniki, Greece; 6835/3 April 2019). Written consent was obtained by all participants.

\section{Conflict of Interest}

None declared.

\section{Acknowledgments}

The authors thank Konstantinos Konstantinou, MD, and "MyLab" Medical Laboratories (Thessaloniki, Greece) for editing and conducting the PCR analysis. 


\section{References}

1 Frencken JE, Sharma P, Stenhouse L, Green D, Laverty D, Dietrich T. Global epidemiology of dental caries and severe periodontitis - a comprehensive review. J Clin Periodontol 2017;44(Suppl 18):S94-S105

2 Tonetti MS, Jepsen S, Jin L, Otomo-Corgel J. Impact of the global burden of periodontal diseases on health, nutrition and wellbeing of mankind: a call for global action. J Clin Periodontol 2017;44(5):456-462

3 Nair S, Faizuddin M, Dharmapalan J. Role of autoimmune responses in periodontal disease. Autoimmune Dis 2014;2014:596824

4 Cekici A, Kantarci A, Hasturk H. Van Dyke TE. Inflammatory and immune pathways in the pathogenesis of periodontal disease. Periodontol 2000 2014;64(1):57-80

5 Socransky SS, Haffajee AD. Dental biofilms: difficult therapeutic targets. Periodontol 2000 2002;28:12-55

6 Bui FQ Almeida-da-Silva CL, Huynh B, et al. Association between periodontal pathogens and systemic disease. Biomed J 2019;42(1):27-35

7 Ansari S, Yamaoka Y. Survival of Helicobacter pylori in gastric acidic territory. Helicobacter 2017;22(4):e12386

8 Zamani M, Ebrahimtabar F, Zamani V, et al. Systematic review with meta-analysis: the worldwide prevalence of Helicobacter pylori infection. Aliment Pharmacol Ther 2018;47(7):868-876

9 Watari J, Chen N, Amenta PS, et al. Helicobacter pylori associated chronic gastritis, clinical syndromes, precancerous lesions, and pathogenesis of gastric cancer development. World J Gastroenterol 2014;20(18):5461-5473

10 Bravo D, Hoare A, Soto C, Valenzuela MA, Quest AF. Helicobacter pylori in human health and disease: Mechanisms for local gastric and systemic effects. World J Gastroenterol 2018;24(28):3071-3089

11 Taş DA, Yakar T, Sakalli H, Serin E. Impact of Helicobacter pylori on the clinical course of recurrent aphthous stomatitis. J Oral Pathol Med 2013;42(1):89-94

12 Adler I, Denninghoff VC, Alvarez MI, Avagnina A, Yoshida R, Elsner B. Helicobacter pylori associated with glossitis and halitosis. Helicobacter 2005;10(4):312-317

13 Attia EA, Abdel Fattah NS, Abdella HM. Upper gastrointestinal findings and detection of Helicobacter pylori in patients with oral lichen planus. Clin Exp Dermatol 2010;35(4):355-360

14 Chey WD, Leontiadis GI, Howden CW, Moss SF. ACG clinical guideline: treatment of Helicobacter pylori infection. Am J Gastroenterol 2017;112(2):212-239

15 Anand PS, Kamath KP, Anil S. Role of dental plaque, saliva and periodontal disease in Helicobacter pylori infection. World J Gastroenterol 2014;20(19):5639-5653

16 Dye BA, Kruszon-Moran D, McQuillan G. The relationship between periodontal disease attributes and Helicobacter pylori infection among adults in the United States. Am J Public Health 2002;92(11):1809-1815

17 Papapanou PN, Sanz M, Buduneli N, et al. Periodontitis: consensus report of workgroup 2 of the 2017 world workshop on the classification of periodontal and peri-implant diseases and conditions. J Periodontol 2018;89(Suppl 1):S173-S182

18 McHugh ML. Interrater reliability: the kappa statistic. Biochem Med (Zagreb) 2012;22(3):276-282

19 Malfertheiner P, Megraud F, O'Morain CA, et al; European Helicobacter and Microbiota Study Group and Consensus panel. Management of Helicobacter pylori infection-the Maastricht V/Florence consensus report. Gut 2017;66(1):6-30

20 Cohen J, Statistical Power Analysis for the Behavioral Sciences. Revised ed. New York, NY: Academic Press; 2013 14-17

21 Kignel S, de Almeida Pina F, André EA, Alves Mayer MP, Birman EG. Occurrence of Helicobacter pylori in dental plaque and saliva of dyspeptic patients. Oral Dis 2005;11(1):17-21
22 Silva DG, Stevens RH, Macedo JMB, et al. Presence of Helicobacter pylori in supragingival dental plaque of individuals with periodontal disease and upper gastric diseases. Arch Oral Biol 2010;55(11):896-901

23 Hardo PG, Tugnait A, Hassan F, et al. Helicobacter pylori infection and dental care. Gut 1995;37(1):44-46

24 Silva Rossi-Aguiar VP, Navarro-Rodriguez T, Mattar R, et al. Oral cavity is not a reservoir for Helicobacter pylori in infected patients with functional dyspepsia. Oral Microbiol Immunol 2009;24(3):255-259

25 Asikainen S, Chen C, Slots J. Absence of Helicobacter pylori in subgingival samples determined by polymerase chain reaction. Oral Microbiol Immunol 1994;9(5):318-320

26 Goosen C, Theron J, Ntsala M, et al. Evaluation of a novel heminested PCR assay based on the phosphoglucosamine mutase gene for detection of Helicobacter pylori in saliva and dental plaque. J Clin Microbiol 2002;40(1):205-209

27 Valadan Tahbaz S, Yadegar A, Amirmozafari N, Yaghoobee S, Ehsani Ardakani MJ, Zojaji H. Occurrence of Helicobacter pylori and its major virulence genotypes in dental plaque samples of patients with chronic periodontitis in Iran. Gastroenterol Hepatol Bed Bench 2017;10(Suppl 1):S70-S78

28 Song Q, Lange T, Spahr A, Adler G, Bode G. Characteristic distribution pattern of Helicobacter pylori in dental plaque and saliva detected with nested PCR. J Med Microbiol 2000;49(4):349-353

29 YeeJK. Helicobacter pylori colonization of the oral cavity: a milestone discovery. World J Gastroenterol 2016;22(2):641-648

30 Okuda K, Kimizuka R, Katakura A, Nakagawa T, Ishihara K. Ecological and immunopathological implications of oral bacteria in Helicobacter pylori-infected disease. J Periodontol 2003;74(1):123-128

31 Czesnikiewicz-Guzik M, Bielanski W, Guzik TJ, Loster B, Konturek SJ. Helicobacter pylori in the oral cavity and its implications for gastric infection, periodontal health, immunology and dyspepsia. J Physiol Pharmacol 2005;56(Suppl 6):77-89

32 Nisha KJ, Nandakumar K, Shenoy KT, Janam P. Periodontal disease and Helicobacter pylori infection: a community-based study using serology and rapid urease test. J Investig Clin Dent 2016;7(1):37-45

33 Al Asqah $\mathrm{M}$, Al Hamoudi $\mathrm{N}$, Anil $\mathrm{S}$, Al Jebreen $\mathrm{A}$, Al-Hamoudi WK. Is the presence of Helicobacter pylori in dental plaque of patients with chronic periodontitis a risk factor for gastric infection? Can J Gastroenterol 2009;23(3):177-179

34 Engstrand L, Nguyen AM, Graham DY, el-Zaatari FA. Reverse transcription and polymerase chain reaction amplification of rRNA for detection of Helicobacter species. J Clin Microbiol 1992;30(9):2295-2301

35 Momtaz H, Souod N, Dabiri H, Sarshar M. Study of Helicobacter pylorigenotype status in saliva, dental plaques, stool and gastric biopsy samples. World J Gastroenterol 2012;18(17):2105-2111

36 Kabir S. Detection of Helicobacter pylori DNA in feces and saliva by polymerase chain reaction: a review. Helicobacter 2004;9(2):115-123

37 Morales-Espinosa R, Fernandez-Presas A, Gonzalez-Valencia $\mathrm{G}$, et al. Helicobacter pylori in the oral cavity is associated with gastroesophageal disease. Oral Microbiol Immunol 2009;24(6):464-468

38 Rahman L, Rahman A, Bakir A, Muhamadamin R, Khudhur P. DNA detection of Helicobacter pylori in saliva of patients with low salivary pH. Zanco J Med Sci 2020;24:283-290

39 Ren Q, Yan X, Zhou Y, Li WX. Periodontal therapy as adjunctive treatment for gastric Helicobacter pylori infection. Cochrane Database Syst Rev 2016;2:CD009477

40 Bouziane A, Ahid S, Abouqal R, Ennibi O. Effect of periodontal therapy on prevention of gastric Helicobacter pylori recurrence: a systematic review and meta-analysis. J Clin Periodontol 2012;39(12):1166-1173 
41 Zarić S, Bojić B, Janković Lj, et al. Periodontal therapy improves gastric Helicobacter pylori eradication. J Dent Res 2009;88(10):946-950

42 Zheng P, Zhou W. Relation between periodontitis and helicobacter pylori infection. Int J Clin Exp Med 2015;8(9):16741-16744

43 Zahedi L, Jafari E, Torabi Parizi M, et al. The association between oral hygiene and gastric pathology in patients with dyspepsia: a cross-sectional study in Southeast Iran. Middle East J Dig Dis 2017;9(1):33-38

44 Adachi K, Notsu T, Mishiro T, Yoshikawa H, Kinoshita Y. Influence of Helicobacter pylori infection on periodontitis. J Gastroenterol Hepatol 2019;34(1):120-123
45 Heitz-Mayfield LJ. Systemic antibiotics in periodontal therapy. Aust Dent J 2009;54(Suppl 1):S96-S101

46 Rôças IN, Siqueira JF Jr. Searching for Helicobacter pylori and Chlamydia pneumoniae in primary endodontic infections. Eur J Dent 2012;6(2):158-162

47 Ishihara K, Miura T, Ebihara Y, Hirayama T, Kamiya S, Okuda K. Shared antigenicity between Helicobacter pylori and periodontopathic Campylobacter rectus strains. FEMS Microbiol Lett 2001;197(1):23-27

48 Umeda M, Kobayashi H, Takeuchi Y, et al. High prevalence of Helicobacter pylori detected by PCR in the oral cavities of periodontitis patients. J Periodontol 2003;74(1):129-134 